\title{
Increased expression of endoplasmic reticulum stress genes in patients with limited cutaneous Systemic Sclerosis and Pulmonary Arterial Hypertension
}

\author{
Stefania Lennaף,§, Alessandra G. Farinaף, Viktor Martyanov ${ }^{\neq}$, Romy B. Christmann , \\ Tammara A Wood, Harrison W Farber ${ }^{\star}$, Raffaella Scorza§, Michael L. Whitfield ${ }^{\neq}$, Robert \\ Lafyatis", and Maria TrojanowskaI \\ "Arthritis Center, Boston University School of Medicine, Boston, MA 02118 \\ §Referral Center for Systemic Autoimmune Diseases, Fondazione IRCCS Ca' Granda Ospedale \\ Maggiore Policlinico and University of Milan, Milan, Italy \\ *Department of Genetics, Geisel School of Medicine at Dartmouth, Hanover, NH 03755 \\ *Pulmonary Center, Boston University School of Medicine, Boston, MA 02118
}

\begin{abstract}
Objective-Pulmonary arterial hypertension (PAH), a common complication of limited cutaneous systemic sclerosis (lcSSc), is associated with alterations of markers of inflammation and vascular damage in peripheral blood mononuclear cells (PBMCs). Endoplasmic reticulum (ER) stress and unfolded protein response (UPR) have been implicated in autoimmune and inflammatory diseases. The goal of this study was to assess whether markers of ER stress/UPR are present in PBMCs from lcSSc-PAH patients.
\end{abstract}

Methods-PBMCs were purified from healthy controls $(\mathrm{HC}, \mathrm{n}=36)$ and $\mathrm{lcSSc}$ patients, with and without PAH (lcSSc-PAH, n=32; lcSSc-NoPAH, n=34). Gene expression in HC PBMCs stimulated with thapsigargin (TG) was analyzed by DNA microarray. Genes were validated by qPCR in $\mathrm{HC}$ and lcSSc PBMCs.

Results-Several ER stress/UPR genes, including Immunoglobulin-heavy-chain binding protein (BiP), Activating Transcription Factor-4 and -6 (ATF4 and ATF6) and a spliced form of X-box binding protein (XBP1) were upregulated in lcSSc PBMCs, with the highest levels in patients with PAH. TG upregulated Heat shock proteins (HSP) and Interferon-regulated genes in control PBMCs. Selected HSP genes, particularly DNAJB1, and IFN-related genes were also found at significantly elevated levels in PBMCs from lcSSc patients, while IRF4 was significantly decreased. There was a positive correlation between DNAJB1 and severity of PAH disease (PAP) $(\mathrm{r}=0.56, \mathrm{p}<0.05)$ and between ER stress markers and IL-6 levels $(\mathrm{r}=0.53, \mathrm{p}<0.0001)$ in lcSSc PBMCs.

Conclusion-This study demonstrates association between select ER stress/UPR markers and lcSSc-PAH suggesting that ER stress/UPR may contribute to the altered function of circulating immune cells in lcSSc.

Correspondence: Maria Trojanowska, Arthritis Center, Boston University School of Medicine, 72 East Concord St, E-5, Boston, MA02118, Tel: 617-638-4318, Fax: 617-638-5226, trojanme@bu.edu. 


\section{INTRODUCTION}

Pulmonary Arterial Hypertension (PAH) is a complex, multi-factorial condition involving numerous biochemical pathways and cell types resulting in alterations in vascular reactivity, vascular structure, and interactions of the vessel wall with circulating blood elements (1). PAH is a serious and often fatal complication of SSc, occurring primarily in patients with the limited cutaneous form of the disease (lcSSc), and is one of the main causes of morbidity and mortality in these patients (2-4). It has been suggested that autoimmunity/inflammation and systemic vasculopathy could contribute to the development of lcSSc-PAH, but the underlying mechanisms have not been fully elucidated (5). Notably, inflammatory cell infiltrates have been observed in perivascular regions of pulmonary arteries more frequently in SSc-PAH than in other forms of PAH $(6,7)$. The cytokines and chemokines released by these immune cells could contribute to the pathological vessel remodeling in PAH (5).

The Endoplasmic Reticulum (ER) is a multifunctional organelle, which coordinates protein folding, lipid biosynthesis, calcium storage and release. Perturbations that disrupt ER homeostasis lead to ER stress and activation of signaling cascades termed the Unfolded Protein Response (UPR). The main UPR branches include PERK (protein kinase RNA-like ER kinase); IRE1 (inositol requiring protein-1) and ATF6 (activating transcription factor6). Under basal conditions these specialized ER membrane-associated sensor proteins are bound by the ER chaperone BiP (immunoglobulin-heavy-chain binding protein, also known as GRP78) and maintained in an inactive state. Accumulation of misfolded proteins in the ER lumen activates adaptive UPR mechanisms through the release of BiP from the sensor proteins and initiation of specific cellular responses aimed at the restoration of ER homeostasis (8). When activated, PERK oligomerizes and phosphorylates itself and the ubiquitous eukaryotic translation initiation factor 2 alpha (eIF2a), which results in global attenuation of translation thus efficiently reducing the influx of proteins to the ER, while selected mRNAs, including ATF4 (activating transcription factor-4), are preferentially translated. PERK also phosphorylates nuclear erythroid 2 p45-related factor (NRF2) involved in restoration of redox balance (9). The transmembrane kinase-endoribonuclease IRE1, governs splicing of the mRNA encoding X-box binding protein-1 (XBP1), a potent transcription factor involved in regulation of genes controlling ER-associated degradation (ERAD), synthesis of ER chaperones, as well as the phospholipid synthesis required for ER expansion. Because of its regulatory role in protein folding and degradation XBP1 is active during the early cytoprotective phase of ER stress, but its activity declines during prolonged ER stress (10). The third arm of the UPR is regulated by ATF6, which upon release from $\mathrm{BiP}$ translocates to the Golgi where it is cleaved by S1P and S2P proteases. A released Nterminal ATF6 fragment moves to the nucleus and in concert with XBP1 activates ER stress target genes, including BiP (8-10). Chronic or severe ER stress activates UPR-dependent apoptotic death. While not yet fully understood, this process is associated with PERK-eIF2a mediated transcription activation of C/EBP-homologous protein (CHOP; also known as GADD153), which then downregulates expression of the antiapoptotic mediator B cell lymphoma 2 (BCL-2) and upregulates expression of proapoptotic BIM. Importantly, recent studies point to a more complex regulation of the intensity and kinetics of UPR signaling depending on the nature of the cellular stress and input from other cellular pathways (10). Accumulating evidence indicates that ER stress is associated with a range of diseases, including neurological disorders, diabetes, metabolic disease, intestinal inflammation and autoimmunity making ER stress a probable instigator of pathological cell death and dysfunction (11-15). Interestingly, there is also evidence that ER homeostasis is closely related to regulation of inflammatory gene expression. A link between ER stress and the inflammatory response was reported in different experimental models, including endothelial cells and immune cells $(13,16-18)$. Inflammation can be triggered by chronic excess of 
metabolic factors, cytokines, and hormones, and those factors can also trigger ER stress, which can further disrupt metabolic function, leading to more inflammation $(18,19)$.

We have recently reported that ectopic expression of HLA-B35, an antigen associated with SSc in Choctow Indians (20) and SSc-PAH in Italian patients $(21,22)$, led to changes in the expression of genes related to ER stress in cultured endothelial cells (ECs) (23).

Furthermore, activation of the ER stress/UPR pathway correlated with upregulation of interferon-regulated genes and other inflammatory genes in ECs expressing HLA-B35. The goal of this study was to further explore the potential contribution of ER stress/UPR to the pathogenesis of lcSSc-PAH by examining expression levels of the ER stress/UPR genes in PBMCs obtained from patients with lcSSc. Herein, we showed for the first time that expression of selected ER stress/UPR genes is significantly elevated in PBMCs from 1SSc patients, with the highest levels observed in patients with PAH. Importantly, there was a positive correlation between ER stress markers and severity of PAH (PAP) and between ER stress markers and IL-6 levels in lcSSc PBMCs, suggesting that activation of ER stress/ UPR, might drive inflammation in PAH associated with SSc.

\section{MATERIALS and METHODS}

\section{Study Participants}

Subjects included normal healthy controls $(\mathrm{HC}, \mathrm{n}=36)$ and patients with $\mathrm{lcSSc}(\mathrm{n}=66)$, according to criteria established by LeRoy et al. (24). Patients with lcSSc were stratified into those with or without PAH based on echocardiography or right heart catheterization (RHC); all patients designated as PAH were confirmed by RHC. Patients were considered not to have PAH $(n=34)$ if echocardiogram demonstrated a pulmonary artery systolic pressure $<35 \mathrm{~mm} \mathrm{Hg}$ and normal right ventricular size and function. Subjects with mean pulmonary arterial pressure $\geq 25 \mathrm{~mm} \mathrm{Hg}$, a pulmonary capillary wedge pressure (PCWP) $\leq 5$ and a pulmonary vascular resistance $\geq 3$ Wood units by RHC were considered to have PAH $(\mathrm{n}=32)$. LSSc patients with mildly elevated pulmonary capillary wedge pressure (PCWP) $(>15$ to $\leq 8)$ were included in our primary analyses consistent with the REVEAL registry with similar rationale (25). Patients that had a mild increase in PCWP included in the primary analysis all had significantly elevated pulmonary vascular resistance (PVR, see supplemental Table 1), and significant increases in both the pulmonary artery diastolic minus pulmonary capillary wedge pressure (PAd-PCWP) gradient $(>10)$ and the transpulmonary gradient $(>15)$. Thus, each was considered to have PAH by the pulmonary hypertension expert caring for the patient. Patient characteristics are listed in supplemental Table 1.

\section{Peripheral Blood Mononuclear Cell (PBMC) isolation}

Blood was collected from healthy controls and patients in CPTTM tubes designed for onestep cell separation (Becton Dickinson, Mountain View, CA). The sample was then immediately mixed and centrifuged at 1,800 RCF at ambient temperature for 30 minutes. The PBMC cell layer was then transferred to a $15 \mathrm{ml}$ tube, and PBMCs were washed twice with PBS and lysed in RNeasy RLT buffer (Qiagen, Valencia, CA).

Healthy control PBMCs were plated in 6 well plates at $8 \times 10^{5}-1 \times 10^{6}$ cells/well in RPMI supplemented with $10 \%$ FCS and $1 \%$ AA and stimulated with 5 or $10 \mathrm{uM}$ Thapsigargin (TM) for 2, 4, 8, 12 and 24 hours. Per manufacturer's protocol, total RNA was extracted using Qiagen's RNeasy Mini kits, and sent for microarray analysis. 


\section{Microarray hybridization and data analysis}

RNA quality was assessed using the Agilent 2100 Bioanalyzer and RNA 6000 Pico Kit. RNA concentration was measured on a Thermo Scientific NanoDrop 2000 Spectrophotometer. Total RNA (25ng) from each sample was converted to Cy3-CTP (Perkin Elmer) labeled cRNA, and Universal Human Reference (UHR) RNA (Stratagene) was converted to Cy5-CTP (Perkin Elmer) labeled cRNA using the Low Input QuickAmp Labeling Kit (Agilent Technologies) for hybridization as described previously (26).

Labeled cRNA from UHR and sample were co-hybridized to Agilent Whole Human Genome (4×44K) Oligo Microarrays (G4112F), representing 41,000 unique genes and transcripts. Microarrays were scanned using Agilent's Dual Laser High-Resolution C Scanner. These microarray image files were processed using Agilent's Feature Extraction software, which automatically finds and places microarray grids, rejects outlier pixels, accurately determines feature intensities and ratios, flags outlier pixels, and calculates statistical confidences. Data were $\log _{2}$ LOWESS normalized for the Cy5/Cy3 ratio, and data was filtered to select array spots with an intensity 2 fold or greater than the local background in either the $\mathrm{Cy} 3$ or Cy5 channel. All data were multiplied by negative one to convert the $\log _{10}(\mathrm{C} 5 / \mathrm{C} 3)$ ratios to $\log _{2}(\mathrm{Cy} 3 / \mathrm{Cy} 5)$ ratios. Probes missing more than $20 \%$ of the data across all arrays were omitted from further analysis. The time course was time zero transformed by subtracting the mean of the zero time points from all other time points.

Gene expression data were organized by average linkage hierarchical clustering using Cluster 3.0 and filtered using 2 arrays, 2 -fold cutoff. That resulted in 3,746 probes. This subset was visualized using Java TreeView and partitioned into 1,582 probes upregulated and 2,164 probes downregulated. Agilent probe IDs were converted to Ensembl gene IDs via g:Convert, a part of $\mathrm{g}$ :Profiler (27). 1,582 upregulated probes were converted to 1,488 unique gene IDs (94.1\% conversion rate) and 2,164 downregulated probes were converted to 1,891 unique gene IDs (87.4\% conversion rate). Sets of Ensembl gene IDs were analyzed for functional enrichment via $\mathrm{g}$ :GOSt, another component of $\mathrm{g}$ :Profiler, in the following annotation categories: Gene Ontology (GO) (28) - biological process (BP), molecular function (MF) and cellular component (CC) and pathways; KEGG (29) and REACTOME (30). Specific functional terms (with the number of annotated genes in the genome below $1,000)$ were selected in order to account for the biologically informative categories.

\section{Quantitative Real-time PCR}

Real-time RT-PCR was performed using IQ ${ }^{\mathrm{TM}}$ SYBR Green Supermix (Bio-Rad) and $\mathrm{MyiQ}^{\mathrm{TM}}$ Single-Color Real-Time PCR Detection System (Bio-Rad). The amount of template used in the PCR reactions was cDNA corresponding to $200 \mathrm{ng}$ reverse-transcribed total RNA. DNA polymerase was first activated at $95^{\circ} \mathrm{C}$ for $3 \mathrm{~min}$., denatured at $95^{\circ} \mathrm{C}$ for $30 \mathrm{sec}$., and annealed/extended at $61^{\circ} \mathrm{C}$ for $30 \mathrm{sec}$., for 40 cycles according to the manufacturer's protocol. Expression of the housekeeping gene $\beta$-actin served as an internal positive control in each assay performed. After measurement of the relative fluorescence intensity for each sample, the amount of each mRNA transcript was expressed as a threshold cycle (ct) value. The primers are available upon request.

\section{Statistical analysis}

Comparisons of RT-PCR expression were analyzed by Student's $t$-test. Correlations were tested by Pearson's correlation coefficient 


\section{RESULTS}

\section{ER stress and UPR genes are upregulated in PBMCs from IcSSc patients}

Subjects selected for this study included patients with lcSSc who were recently characterized in a study examining expression of PAH biomarkers (31), as well as an additional lcSSc patients with PAH and lcSSc patients without PAH (see supplemental Table 1). PBMC samples from a total of 34 ISSc patients without PAH (lcSSc-NoPAH), 32 1SSc patients with PAH (lcSSc-PAH) and 36 healthy controls (HC) were analyzed for the presence of ER stress/UPR-related genes. The candidate ER stress/UPR genes consisted of molecular chaperone BiP/Grp78, indicator of the onset of the UPR, as well as key regulators of the UPR pathway including transcription factors ATF4, ATF6, and XPB1. Compared with healthy control subjects, PBMCs from lcSSc -NoPAH patients demonstrated significantly higher expression of $\mathrm{BiP}(\mathrm{p}<0.0001)$. The highest level of $\mathrm{BiP}$ was present in samples from patients with lcSSc-PAH when compared to lcSSc-NoPAH samples $(\mathrm{p}<0.05)$ or HCs $(\mathrm{p}<0.0001)$ (Fig.1). The transcription factors ATF4 and ATF6 were significantly elevated in patients with lcSSc-PAH compared to HCs $(\mathrm{p}<0.001)$ and $1 \mathrm{lSSc}-\mathrm{NoPAH}$ $(\mathrm{p}<0.05)$ for ATF6. XBP1 splicing was increased in lcSSc compared with $\mathrm{HCs}(\mathrm{p}<0.05)$, but no significant difference was observed between lcSSc-NoPAH and lcSSc-PAH. Together, these results demonstrate that activation of selected ER stress/UPR in PBMCs is associated with lcSSc and is progressively increased in patients with PAH.

\section{Thapsigargin upregulates heat shock proteins and interferon-regulated genes in PBMCs}

Microarray analyses were used to further investigate genes regulated by ER stress in PBMCs. PBMCs were isolated from healthy controls and treated with a known ER stress inducer, Thapsigargin (TG), which activates UPR by inhibiting the ER $\mathrm{Ca}^{2+}$ ATPase. The basal expression levels of 3,746 probes were significantly changed in response to TG compared to untreated PBMCs. Analysis of enriched functional terms among the probes with increased and decreased expression showed terms generally associated with immune responses including B cell activation, cytokine production and inflammatory response, among others. Genes with increased expression were enriched in terms related to the endoplasmic reticulum and the unfolded protein response (Supplemental Table 2). Genes with decreased expression showed significant enrichment in functional terms related to homeostasis, lysosome and GTPase signaling (Supplemental Table 3). A complete functional enrichment data is available upon request.

Examination of specific sets of genes deregulated by TG treatment showed increased expression of heat shock proteins (HSP) and IFN-regulated genes (Fig 2). The HSP group consisted of HSP70 (HSP5A, HSP9B) and its co-chaperones, HSP40 isoforms (DNAJB1, DNAJB5, DNAJB11 and DNAJC3), HSP90 (HSPCB) and HSP150/110 (HSPH1). IFN related genes with altered expression included many well-known IFN-regulated genes: IFI44, IFI44L, IFIT1, IFIT2, IFIT3, IFIT5, IFITM3, MX1, IFN alpha-inducible proteins 2 and 3 (GlP2 and G1P3), Interferon-induced guanylate-binding protein 1 (GBP1), IFNinducible RNA dependent protein kinase (PRKR), and IFN regulatory factor 4-1-7 (IRF4, IRF1, IRF7). Upregulation of HSP and representative IFN-regulated genes in PBMCs treated with TG was confirmed by qPCR (data not shown).

\section{DNAJ/HSP40 genes are upregulated in PBMCs from IcSSc-PAH patients}

DNAJ/HSP40 isoforms were among the genes most notably altered in the TG treated PBMCs and were subsequently analyzed in control and patient PBMCs by qRT-PCR. Compared with healthy control subjects, PBMCs from lcSSc-NoPAH patients demonstrated elevated expression of DNAJB1 ( $\mathrm{p}<0.0001)$ with a further increase in lcSSc-PAH $(\mathrm{p}<0.05)$. DNAJB11 was less prominently, but also significantly increased in lcSSc-PAH samples 
(p<0.05), (Fig. 3). Furthermore, expression of DNAJB1 showed the highest positive correlation with pulmonary arterial pressure (PAP) by catheterization of lcSSc patients $(\mathrm{r}=0.56, \mathrm{p}<0.05)$. On the other hand, with the exception of BiP, expression of other HSP70 isoforms, as well as HSP90 and HSP150/110 isoforms was not significantly different between $\mathrm{HC}$ and lcSSc (with or without PAH) PBMCs (data no shown), suggesting a distinct association of HSP40 with lcSSc.

\section{IFN-regulated genes are altered in PBMCs from IcSSc-PAH patients}

The presence of "interferon signature" in SSc PBMCs, including patients with lcSSc has previously been reported $(31,32)$. From the cluster of the IFN-regulated genes induced by TG in HC PBMCs (Fig. 2), we selected several representative genes that were not previously validated in patient PBMCs, including IFIT1, IFIT2, IFITM1, and IRF4 for the qPCR analysis in PBMCs. Consistent with previous studies IFIT1, IFIT2, and IFITM1 were expressed at a significantly higher level in patients with lcSSc-NoPAH vs healthy controls (Fig. 4) but did not show a progressive increase in lcSSc-PAH patients compared to lcSScNoPAH. Interestingly, IRF4 was significantly downregulated in lcSSc vs HC (HC vs lcSScNoPAH, $\mathrm{p}<0.0001, \mathrm{HC}$ vs lcSSc-PAH, $\mathrm{p}<0.001)$. Expression of other IRF genes, including IRF5, was not significantly changed.

To further investigate whether endogenously produced interferon was contributing to the UPR-mediated upregulation of IFN-related genes, HC PBMCs were treated for 24 hours with TG (5pM) or IFNa (250U, used as control) in combination with B18R, a decoy receptor for type I Interferon. Selected IFN-induced genes were analyzed by qPCR. IFNainduced IFIT1, IFITM1, MX1, CXCL10, IRF7 and IRF4 were abrogated by B18R. Although, blockade of IFN also attenuated upregulation of those genes in response to TG, the inhibitory effects were less potent, possibly suggesting contribution of the IFNindependent pathways (Fig. 5). Interestingly, while IRF4 was only moderately upregulated by IFNa, it was very potently upregulated by TG. Unexpectedly, B18R further potentiated stimulation of IRF4 by TG, suggesting that IFN may both positively and negatively regulate IRF4 expression depending on the activation status of other signaling pathways.

\section{ER stress/UPR correlates with increased expression levels of pro-inflammatory cytokines}

Elevated expression levels of inflammatory mediators, including IL-6, IL-1 $\beta$ and IL-13 have been previously reported in PBMCs from lcSSc patients (33-35). mRNA expression of IL-6 was determined in PBMC samples used in this study. In agreement with previous findings IL-6 mRNA levels were significantly elevated in lcSSc vs healthy control $(\mathrm{p}<0.005)$ PBMCs, with the highest levels in lcSSc-PAH PBMCs. Notably, there was a positive correlation $(r=0.53, p<0.0001)$ between mRNA expression of IL-6 and BiP in PBMC samples from patients with ISSc (Fig. 6). Similar correlations were observed when mRNA level of DNAJB1 were compared with IL-6 levels (data not shown). These results suggest that ER stress/UPR could contribute to increased inflammation in patients with lcSSc.

\section{DISCUSSION}

This study characterized expression of ER stress and UPR related genes in PBMCs obtained from a well-characterized cohort of lcSSc patients with and without evidence of PAH, as well as healthy control subjects. We demonstrate that lcSSc PBMCs express significantly higher levels of genes representing all three branches of the UPR. Notably, BiP, ATF6, ATF4, as well as chaperone DNAJB1 showed the highest levels of expression in patients with PAH. On the other hand, spliced XBP1 was only moderately elevated in lcSSc patients with no evidence of further upregulation in patients with $\mathrm{PAH}$. A less pronounced activation of the XBP1 pathway may indicate a chronic nature of the ER stress in patients with PAH 
(10). There was a significant correlation between expression of DNAJB1 and pulmonary arterial pressure, as well as $\mathrm{BiP}$ and IL-6 expression suggesting that chronic UPR may contribute to increased inflammation in lcSSc through activation of the NF- $\kappa$ B and AP-1 pathways (12).

The current study further expands the analyses of biomarkers in lcSSc PBMCs $(32,36)$. Similar to the previously observed pattern of gene expression in the "biomarker cluster" (IL3RA1, CCR1), selected ER chaperons, including BiP and DNAJB1, showed an increased expression in patients with lcSSc-NoPAH with the highest level of expression in patients with PAH. Expression of DNAJB, as well as BiP correlated strongly with PAP by rightheart catheterization. An earlier study by Christmann et al showed a strong association of the macrophage alternative activation marker gene, MRC1, with PAP, (32), but ER stress genes only weakly correlated with MRC1 ( $\mathrm{r}=0.32, \mathrm{p}<0.05$ for $\mathrm{MRC} 1$ and $\mathrm{BiP})$ and did not correlate with IL13RA1 but, instead correlated strongly with IL-6 $(0.53, \mathrm{p}<0.0001$ for BiP and IL-6). These results suggest heterogeneous mechanisms involved in worsening PAP in patients with lcSSc. Further studies are needed to determine whether increased levels of ER stress markers in lcSSc patients results from the changes in a specific subset of cells, e.g. monocytes or dendritic cells, or whether a majority of circulating cells contribute to the ER stress signature.

Growing evidence suggests that inflammation contributes to the development and/or progression of PAH. Plexiform lesions often contain lymphocytes, macrophages and mast cells, and antibody-complement deposits have been associated with PAH $(5,7)$.

Furthermore, elevated expression levels of several inflammatory mediators have been observed in plasma of PAH patients (33). In particular, IL-6 has been implicated in the pathogenesis of PAH $(32,33)$ and increased levels of IL-6 have been reported in lcSSc-PAH patients $(34,36)$. Consistent with those earlier reports, we found elevated levels of IL-6 mRNA levels in lcSSc PBMCs, with the highest levels in lcSSc-PAH samples. Furthermore there was a positive and significant correlation between BiP and IL-6 mRNA expression levels, consistent with the possibility that ER stress may function as an endogenous inducer of inflammation. Interestingly, several of the ER stress/UPR genes showed variable expression in healthy individuals. A recent study by Dombroski et al (37) that characterized ER stress/UPR in human B cells obtained from healthy individuals have also found an extensive individual variability in expression of the ER stress/UPR related genes in response to the ER stress-inducing agents. Many of these variable genes were the known disease linked genes, suggesting a mechanistic link between ER function and human disease. Indeed, genetic polymorphisms in UPR genes have been functionally linked to inflammatory bowel disease and asthma $(38,39)$. Relevant to these findings, we have previously shown that HLA-B35, which is associated with increased risk for developing PAH in Italian SSc patients $(21,22)$ induced ER stress-mediated upregulation of endothelin 1, proinflammatory cytokines, and IFN-related genes in human microvascular endothelial cells (23). However, factors contributing to the induction of ER stress/UPR in patients with SSc are currently unknown.

Analyses of PBMCs subjected to thapsigargin-mediated ER stress in vitro revealed upregulation of a large number of IFN-regulated genes consistent with previous studies that demonstrated induction of IFNa by TG in mouse macrophages (RAW 264.7 cells) (40). Furthermore, priming of RAW 264.7 cells with TG greatly potentiated their responses to TLR4 and TLR3 ligands and this synergistic interaction was completely dependent on XBP-1 (40). In agreement with the previous reports $(32,34,35)$, we have also observed increased expression of IFN-related genes, including IFIT1, IFIT2, and IFITM1 that correlated with increased XBP-1 splicing in our lcSSc patient cohort, suggesting that ER stress may contribute, among other factors, to the "interferon signature" in lcSSc patients. 
Consistent with this conclusion, we found that upregulation of IFN-regulated genes by TG in PBMCs was in part dependent on activation of the endogenous type I IFN. Unexpectedly, while both IFNa and TG induced IRF4 in HC PBMCs, there was a significant decrease of IRF4 expression in lcSSc-NoPAH and lcSSc-PAH PBMCs compared to healthy individuals, suggesting that ER stress and/or IFN-dependent pathways may not be involved in the downregulation of IRF4 in lcSSc patients. Given the prominent role of IRF4 in regulation of the immune response, in the future it would be important to focus on the mechanisms involved in its dysregulation in lcSSc. IRF4 is expressed in both T and B cells and plays an important role in mature CD4+ T cell function (rev. in (41). Interestingly, a lack of IRF4 results in enhanced IFNa production under TH2 conditions (41). Additional studies revealed that peritoneal macrophages isolated from Irf4-deficient mice showed markedly enhanced induction of IL-6 and IL-12p40 in response TLR4, TLR7 or TLR9 stimulation (42). Functionally, IRF4 was shown to compete with IRF5, but not IRF7 for MyD88 interaction (42). Since IRF5 expression levels were not changed in lcSSc PBMCs analyzed in this study (data not shown), lower levels of IRF4 may alter IRF5 signaling in this group of patients. Importantly, an IRF5 polymorphism was identified as one of the susceptibility loci associated with SSc in a recent SSc-genome wide association study (43). Together, these studies suggest that low expression levels of IRF4 may contribute to the enhanced cytokine production in lcSSc patients.

In conclusion, we demonstrate increased expression of selected UPR genes in PBMCs from patients with lcSSc. Since activation of UPR may result from a variety of factors that disturb cellular homeostasis, additional studies will be required to investigate specific factors contributing to this process in lcSSc. It may be relevant that viral infections have been associated with induction of UPR, as well as the interferon response (44), and since herpesviruses have been linked to SSc pathogenesis (45-47), it may suggest a possible role for a herpesvirus in activation of the UPR response in lcSSc. Regardless of the initial stimulus, activation of the UPR may signal disease worsening and selected ER stress/UPR genes may serve as markers of disease progression in patients with lcSSc. Interestingly, activation of ER stress/UPR markers was recently demonstrated in monocytes from patients with type 2 diabetes and was implicated in attenuation of the response to TLR agonists (48). It may also be relevant that an altered TLR-mediated induction of proinflammatory cytokines was observed in dendritic cells from patients with SSc, including lcSSc (49). Future studies should determine whether ER stress/UPR alter the function of immune cells in patients with lcSSc.

\section{Supplementary Material}

Refer to Web version on PubMed Central for supplementary material.

\section{Acknowledgments}

This work was supported by a grant from GILS (Gruppo Italiano per la Lotta alla Sclerodermia) to SL, Scleroderma Foundation grants (AGF, HWF, MT) and P50 AR060780 (RL, HWF, MT, MLW).

\section{References}

1. Humbert M, Morrell NW, Archer SL, Stenmark KR, MacLean MR, Lang IM, et al. Cellular and molecular pathobiology of pulmonary arterial hypertension. J Am Coll Cardiol. 2004; 43(12 Suppl S):13S-24S. [PubMed: 15194174]

2. Le Pavec J, Humbert M, Mouthon L, Hassoun PM. Systemic sclerosis-associated pulmonary arterial hypertension. Am J Respir Crit Care Med. 2010; 181(12):1285-1293. [PubMed: 20194816] 
3. MacGregor AJ, Canavan R, Knight C, Denton CP, Davar J, Coghlan J, et al. Pulmonary hypertension in systemic sclerosis: risk factors for progression and consequences for survival. Rheumatology (Oxford). 2001; 40(4):453-459. [PubMed: 11312386]

4. Steen V, Medsger TA Jr. Predictors of isolated pulmonary hypertension in patients with systemic sclerosis and limited cutaneous involvement. Arthritis Rheum. 2003; 48(2):516-522. [PubMed: 12571862]

5. Hassoun PM, Mouthon L, Barbera JA, Eddahibi S, Flores SC, Grimminger F, et al. Inflammation, growth factors, and pulmonary vascular remodeling. J Am Coll Cardiol. 2009; 54(1 Suppl):10-19.

6. Dorfmuller P, Humbert M, Perros F, Sanchez O, Simonneau G, Muller KM, et al. Fibrous remodeling of the pulmonary venous system in pulmonary arterial hypertension associated with connective tissue diseases. Hum Pathol. 2007; 38(6):893-902. [PubMed: 17376507]

7. Tuder RM. Pathology of pulmonary arterial hypertension. Semin Respir Crit Care Med. 2009; 30(4): 376-385. [PubMed: 19634077]

8. Ron D, Walter P. Signal integration in the endoplasmic reticulum unfolded protein response. Nat Rev Mol Cell Biol. 2007; 8(7):519-529. [PubMed: 17565364]

9. Wang S, Kaufman RJ. The impact of the unfolded protein response on human disease. J Cell Biol. 2012; 197(7):857-867. [PubMed: 22733998]

10. Hetz C. The unfolded protein response: controlling cell fate decisions under ER stress and beyond. Nat Rev Mol Cell Biol. 2012; 13(2):89-102. [PubMed: 22251901]

11. Hotamisligil GS. Endoplasmic reticulum stress and atherosclerosis. Nat Med. 2010; 16(4):396399. [PubMed: 20376052]

12. Hotamisligil GS. Endoplasmic reticulum stress and the inflammatory basis of metabolic disease. Cell. 2010; 140(6):900-917. [PubMed: 20303879]

13. Kaser A, Martinez-Naves E, Blumberg RS. Endoplasmic reticulum stress: implications for inflammatory bowel disease pathogenesis. Curr Opin Gastroenterol. 2010; 26(4):318-326. [PubMed: 20495455]

14. Salminen A, Kauppinen A, Suuronen T, Kaarniranta K, Ojala J. ER stress in Alzheimer's disease: a novel neuronal trigger for inflammation and Alzheimer's pathology. J Neuroinflammation. 2009; 6:41. [PubMed: 20035627]

15. Todd DJ, Lee AH, Glimcher LH. The endoplasmic reticulum stress response in immunity and autoimmunity. Nat Rev Immunol. 2008; 8(9):663-674. [PubMed: 18670423]

16. Gargalovic PS, Gharavi NM, Clark MJ, Pagnon J, Yang WP, He A, et al. The unfolded protein response is an important regulator of inflammatory genes in endothelial cells. Arterioscler Thromb Vasc Biol. 2006; 26(11):2490-2496. [PubMed: 16931790]

17. Komura T, Sakai Y, Honda M, Takamura T, Matsushima K, Kaneko S. CD14+ monocytes are vulnerable and functionally impaired under endoplasmic reticulum stress in patients with type 2 diabetes. Diabetes. 2010; 59(3):634-643. [PubMed: 19959758]

18. Zhang K, Kaufman RJ. From endoplasmic-reticulum stress to the inflammatory response. Nature. 2008; 454(7203):455-462. [PubMed: 18650916]

19. Kaser A, Blumberg RS. Endoplasmic reticulum stress and intestinal inflammation. Mucosal Immunol. 2010; 3(1):11-16. [PubMed: 19865077]

20. Arnett FC, Howard RF, Tan F, Moulds JM, Bias WB, Durban E, et al. Increased prevalence of systemic sclerosis in a Native American tribe in Oklahoma. Association with an Amerindian HLA haplotype. Arthritis Rheum. 1996; 39(8):1362-1370. [PubMed: 8702445]

21. Santaniello A, Salazar G, Lenna S, Antonioli R, Colombo G, Beretta L, et al. HLA-B35 upregulates the production of endothelin-1 in HLA-transfected cells: a possible pathogenetic role in pulmonary hypertension. Tissue Antigens. 2006; 68(3):239-244. [PubMed: 16948645]

22. Scorza R, Caronni M, Bazzi S, Nador F, Beretta L, Antonioli R, et al. Post-menopause is the main risk factor for developing isolated pulmonary hypertension in systemic sclerosis. Ann N Y Acad Sci. 2002; 966:238-246. [PubMed: 12114278]

23. Lenna S, Townsend DM, Tan FK, Kapanadze B, Markiewicz M, Trojanowska M, et al. HLA-B35 upregulates endothelin-1 and downregulates endothelial nitric oxide synthase via endoplasmic reticulum stress response in endothelial cells. J Immunol. 2010; 184(9):4654-4661. [PubMed: 20335527] 
24. LeRoy EC, Black C, Fleischmajer R, Jablonska S, Krieg T, Medsger TA Jr, et al. Scleroderma (systemic sclerosis): classification, subsets and pathogenesis. J Rheumatol. 1988; 15(2):202-205. [PubMed: 3361530]

25. McGoon MD, Krichman A, Farber HW, Barst RJ, Raskob GE, Liou TG, et al. Design of the REVEAL registry for US patients with pulmonary arterial hypertension. Mayo Clin Proc. 2008; 83(8):923-931. [PubMed: 18674477]

26. Milano A, Pendergrass SA, Sargent JL, George LK, McCalmont TH, Connolly MK, et al. Molecular subsets in the gene expression signatures of scleroderma skin. PLoS One. 2008; 3(7):e2696. [PubMed: 18648520]

27. Reimand J, Kull M, Peterson H, Hansen J, Vilo J. g:Profiler--a web-based toolset for functional profiling of gene lists from large-scale experiments. Nucleic acids research. 2007; 35(Web Server issue):W193-W200. [PubMed: 17478515]

28. Ashburner M, Ball CA, Blake JA, Botstein D, Butler H, Cherry JM, et al. Gene ontology: tool for the unification of biology. The Gene Ontology Consortium. Nat Genet. 2000; 25(1):25-29. [PubMed: 10802651]

29. Ogata H, Goto S, Sato K, Fujibuchi W, Bono H, Kanehisa M. KEGG: Kyoto Encyclopedia of Genes and Genomes. Nucleic acids research. 1999; 27(1):29-34. [PubMed: 9847135]

30. Joshi-Tope G, Gillespie M, Vastrik I, D'Eustachio P, Schmidt E, de Bono B, et al. Reactome: a knowledgebase of biological pathways. Nucleic acids research. 2005; 33(Database issue):D428D432. [PubMed: 15608231]

31. Pendergrass SA, Hayes E, Farina G, Lemaire R, Farber HW, Whitfield ML, et al. Limited systemic sclerosis patients with pulmonary arterial hypertension show biomarkers of inflammation and vascular injury. PLoS One. 5(8)

32. Christmann RB, Hayes E, Pendergrass S, Padilla C, Farina G, Affandi AJ, et al. Interferon and alternative activation of monocyte/macrophages in systemic sclerosis-associated pulmonary arterial hypertension. Arthritis Rheum. 63(6):1718-1728. [PubMed: 21425123]

33. Gourh P, Arnett FC, Assassi S, Tan FK, Huang M, Diekman L, et al. Plasma cytokine profiles in systemic sclerosis: associations with autoantibody subsets and clinical manifestations. Arthritis Res Ther. 2009; 11(5):R147. [PubMed: 19799786]

34. Tan FK, Zhou X, Mayes MD, Gourh P, Guo X, Marcum C, et al. Signatures of differentially regulated interferon gene expression and vasculotrophism in the peripheral blood cells of systemic sclerosis patients. Rheumatology (Oxford). 2006; 45(6):694-702. [PubMed: 16418202]

35. York MR, Nagai T, Mangini AJ, Lemaire R, van Seventer JM, Lafyatis R. A macrophage marker, Siglec-1, is increased on circulating monocytes in patients with systemic sclerosis and induced by type I interferons and toll-like receptor agonists. Arthritis Rheum. 2007; 56(3):1010-1020. [PubMed: 17328080]

36. Pendergrass SA, Hayes E, Farina G, Lemaire R, Farber HW, Whitfield ML, et al. Limited systemic sclerosis patients with pulmonary arterial hypertension show biomarkers of inflammation and vascular injury. PLoS One. 2010; 5(8):e12106. [PubMed: 20808962]

37. Dombroski BA, Nayak RR, Ewens KG, Ankener W, Cheung VG, Spielman RS. Gene expression and genetic variation in response to endoplasmic reticulum stress in human cells. Am J Hum Genet. 2010; 86(5):719-729. [PubMed: 20398888]

38. Cantero-Recasens G, Fandos C, Rubio-Moscardo F, Valverde MA, Vicente R. The asthmaassociated ORMDL3 gene product regulates endoplasmic reticulum-mediated calcium signaling and cellular stress. Hum Mol Genet. 19(1):111-121. [PubMed: 19819884]

39. Kaser A, Lee AH, Franke A, Glickman JN, Zeissig S, Tilg H, et al. XBP1 links ER stress to intestinal inflammation and confers genetic risk for human inflammatory bowel disease. Cell. 2008; 134(5):743-756. [PubMed: 18775308]

40. Smith JA, Turner MJ, DeLay ML, Klenk EI, Sowders DP, Colbert RA. Endoplasmic reticulum stress and the unfolded protein response are linked to synergistic IFN-beta induction via X-box binding protein 1. Eur J Immunol. 2008; 38(5):1194-1203. [PubMed: 18412159]

41. Biswas PS, Bhagat G, Pernis AB. IRF4 and its regulators: evolving insights into the pathogenesis of inflammatory arthritis? Immunol Rev. 2010; 233(1):79-96. [PubMed: 20192994] 
42. Negishi H, Ohba Y, Yanai H, Takaoka A, Honma K, Yui K, et al. Negative regulation of Toll-likereceptor signaling by IRF-4. Proc Natl Acad Sci U S A. 2005; 102(44):15989-15994. [PubMed: 16236719]

43. Radstake TR, Gorlova O, Rueda B, Martin JE, Alizadeh BZ, Palomino-Morales R, et al. Genomewide association study of systemic sclerosis identifies CD247 as a new susceptibility locus. Nat Genet. 2010; 42(5):426-429. [PubMed: 20383147]

44. He B. Viruses, endoplasmic reticulum stress, and interferon responses. Cell Death Differ. 2006; 13(3):393-403. [PubMed: 16397582]

45. Arnson Y, Amital H, Guiducci S, Matucci-Cerinic M, Valentini G, Barzilai O, et al. The role of infections in the immunopathogensis of systemic sclerosis--evidence from serological studies. Ann N Y Acad Sci. 2009; 1173:627-632. [PubMed: 19758208]

46. Pandey JP, LeRoy EC. Human cytomegalovirus and the vasculopathies of autoimmune diseases (especially scleroderma), allograft rejection, and coronary restenosis. Arthritis Rheum. 1998; 41(1):10-15. [PubMed: 9433864]

47. Vaughan JH, Shaw PX, Nguyen MD, Medsger TA Jr, Wright TM, Metcalf JS, et al. Evidence of activation of 2 herpesviruses, Epstein-Barr virus and cytomegalovirus, in systemic sclerosis and normal skins. J Rheumatol. 2000; 27(3):821-823. [PubMed: 10743836]

48. Komura T, Sakai Y, Honda M, Takamura T, Matsushima K, Kaneko S. CD14+ monocytes are vulnerable and functionally impaired under endoplasmic reticulum stress in patients with type 2 diabetes. Diabetes. 59(3):634-643. [PubMed: 19959758]

49. van Bon L, Popa C, Huijbens R, Vonk M, York M, Simms R, et al. Distinct evolution of TLRmediated dendritic cell cytokine secretion in patients with limited and diffuse cutaneous systemic sclerosis. Ann Rheum Dis. 69(8):1539-1547. [PubMed: 20498209] 

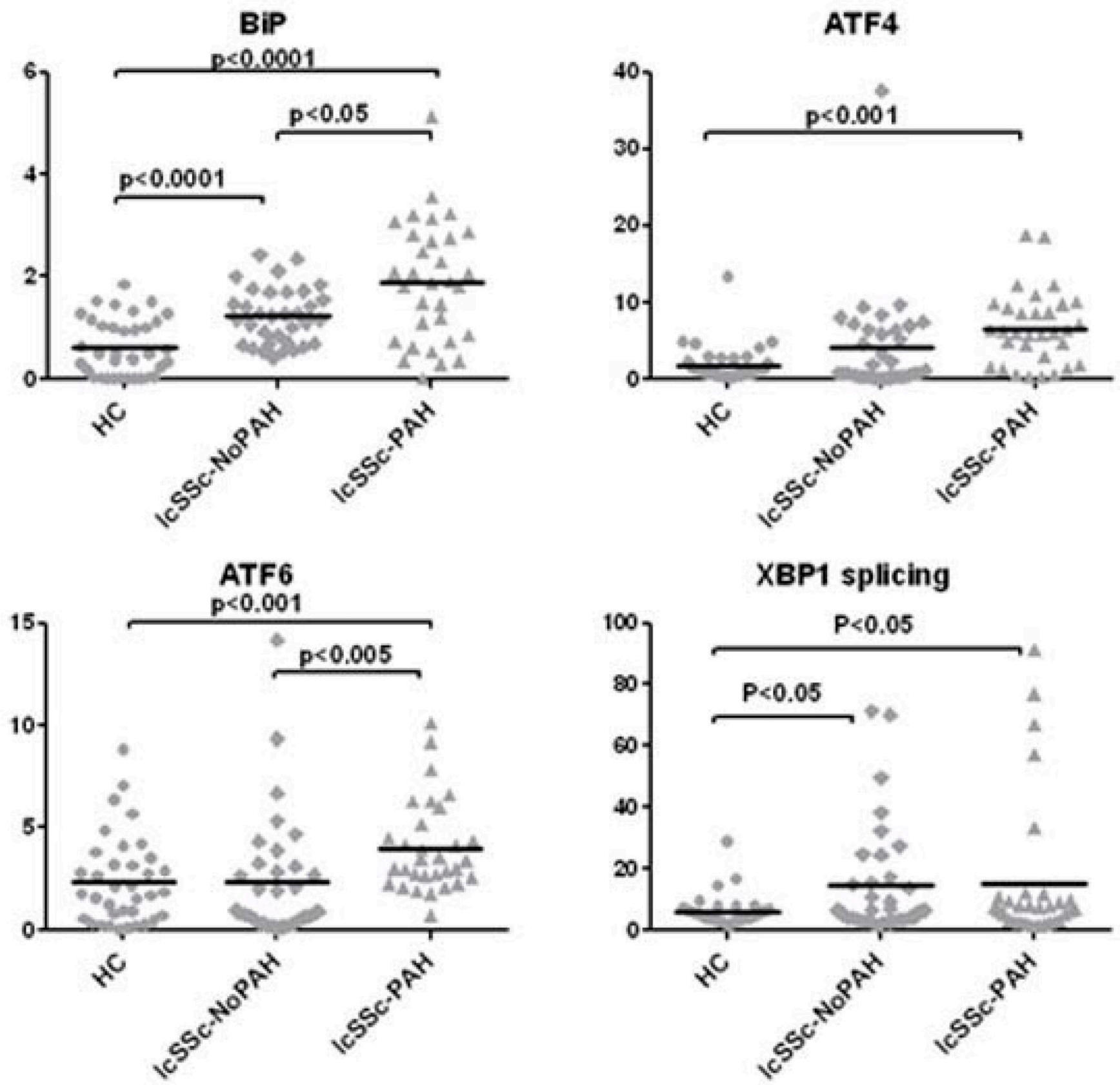

Figure 1. Elevated mRNA expression of selected ER stress/UPR markers in patients with lcSSc PBMCs were isolated from HC $(n=36), 1 c S S c-N o P A H ~(n=34)$ and lcSSc-PAH $(n=32)$. mRNA levels of BiP and ATF4 (top panel), ATF6 and XBP1 splicing (bottom panel) were measured by qPCR. Expression of the housekeeping gene $\beta$-actin served as an internal positive control. Data are expressed as the fold-change normalized to mRNA expression in a single HC sample. Each data point represents a single subject; horizontal lines show the mean. 


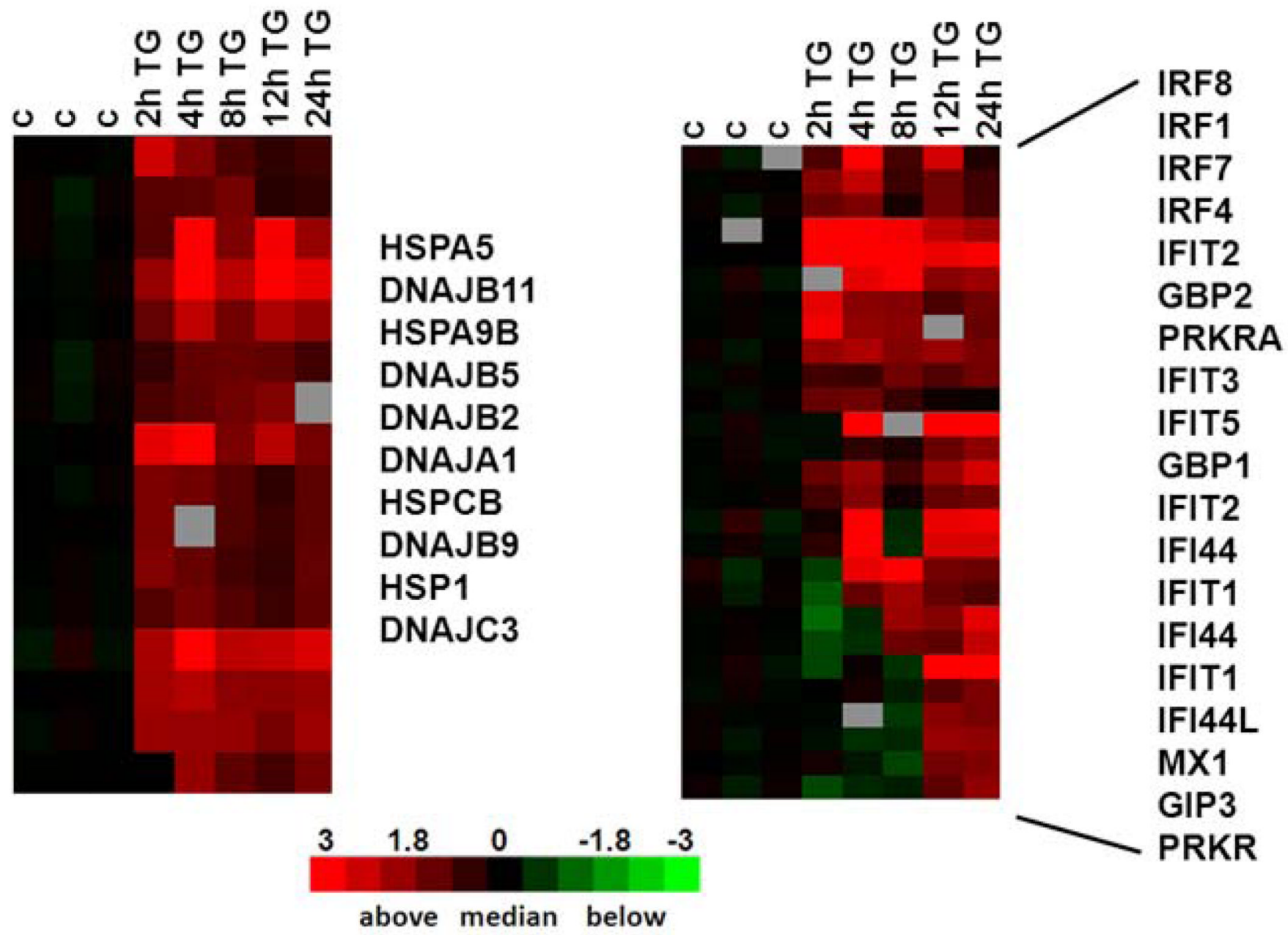

Figure 2. HSP and IFN-regulated gene expression in healthy control PBMCs treated with thapsigargin

Heat map showing the expression of genes clustered using average linkage, hierarchical, and supervised clustering. Left, Cluster of HSP genes, right, Cluster of IFN-regulated genes. Values above the mean expression level of each gene are indicated in red and below the mean are indicated in green. 
DNAJB1

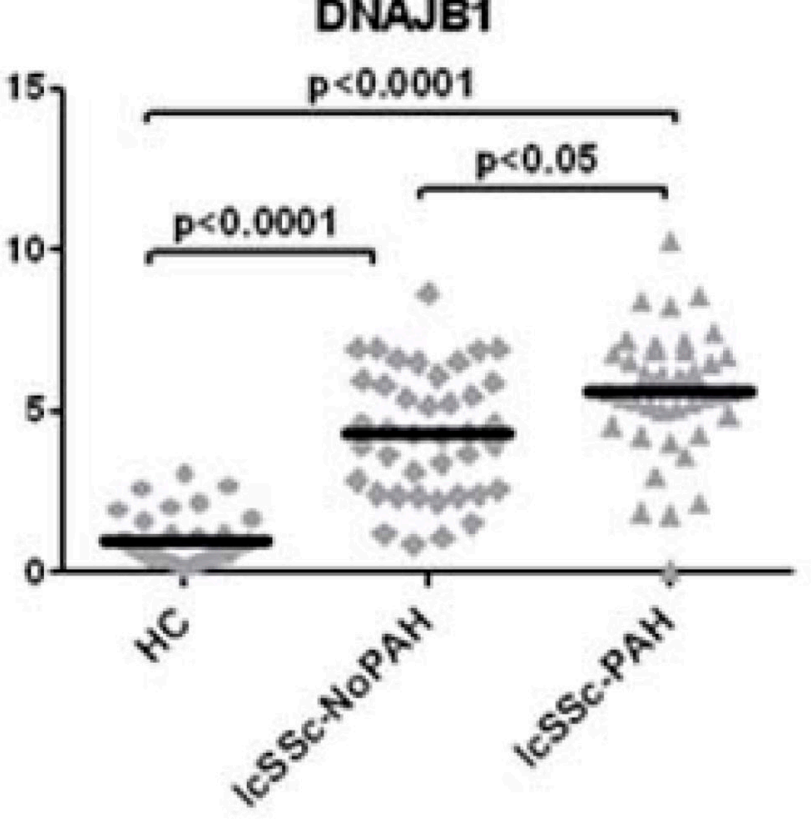

DNAJB11

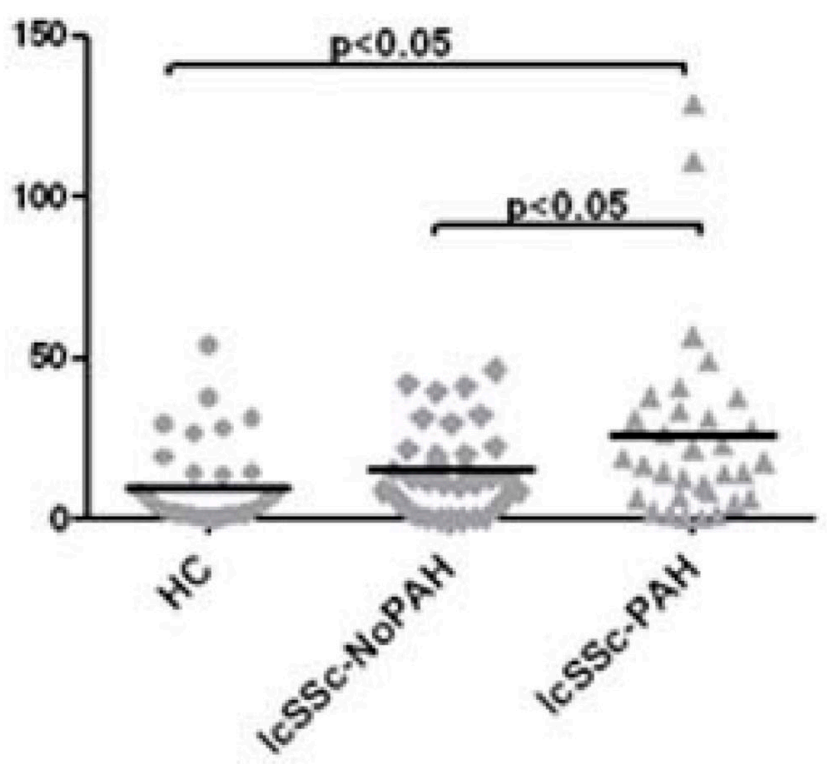

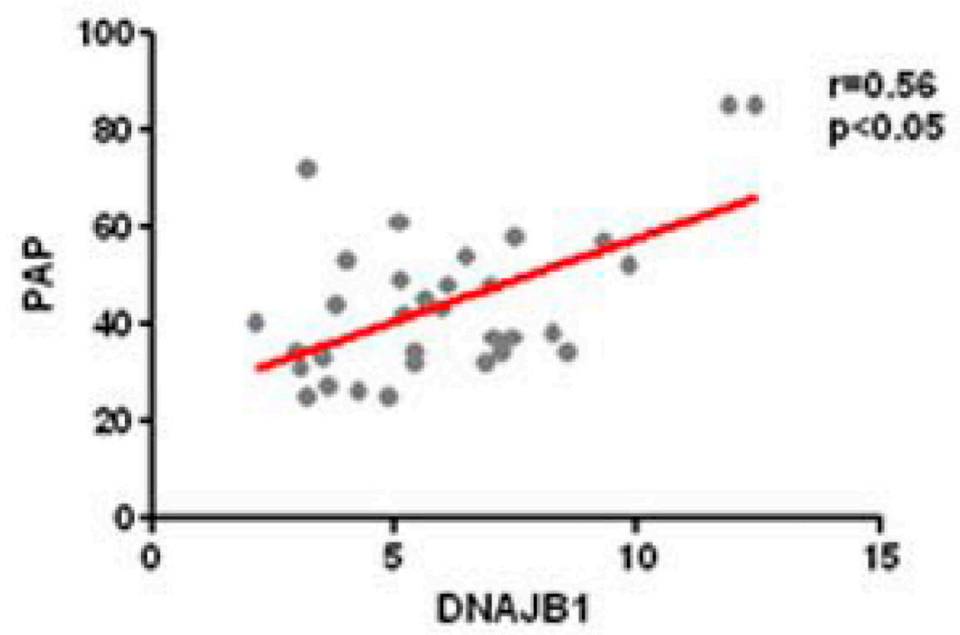

Figure 3. Elevated mRNA expression of selected DNAJ/HSP40 in patients with lcSSc PBMCs were isolated from HC $(n=36), 1 c S S c-N o P A H ~(n=34)$ and lcSSc-PAH $(n=32)$. mRNA levels of DNAJ/HSP40 ( DNAJB1, DNAJB11) were measured by qPCR. Expression of the housekeeping gene $\beta$-actin served as an internal positive control. Data are expressed as the fold-change normalized to mRNA expression in a single HC sample. Each data point represents a single subject; horizontal lines show the mean. Right bottom panel depicts linear regression analysis of the relationship between expression of PAP and DNAJB1 in PBMCs from lcSSc-PAH patients. 
IFIT 1

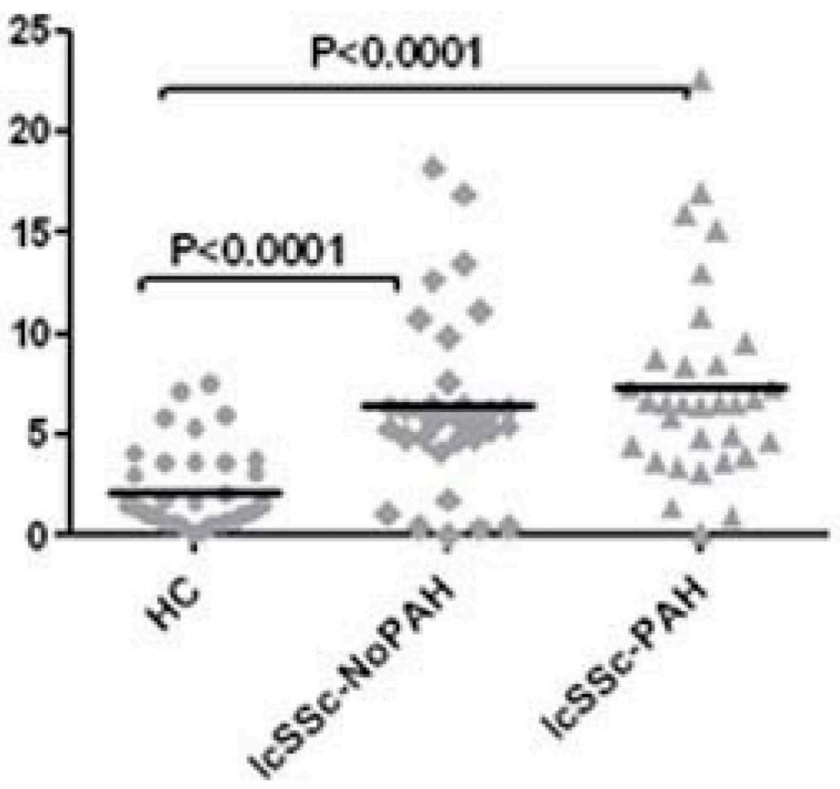

IFITM1

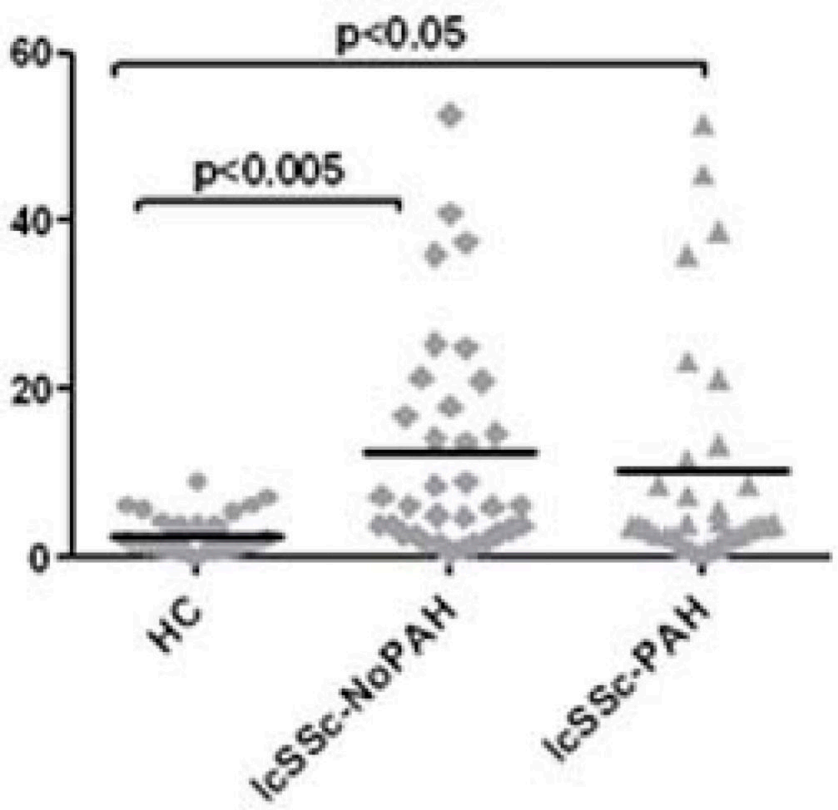

IFIT 2

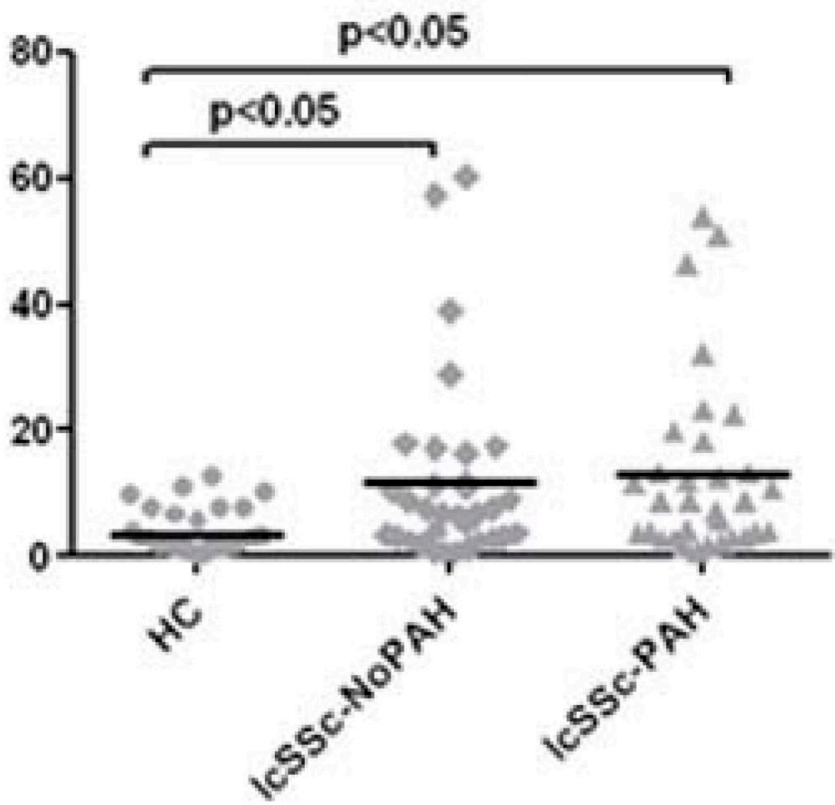

IRF4

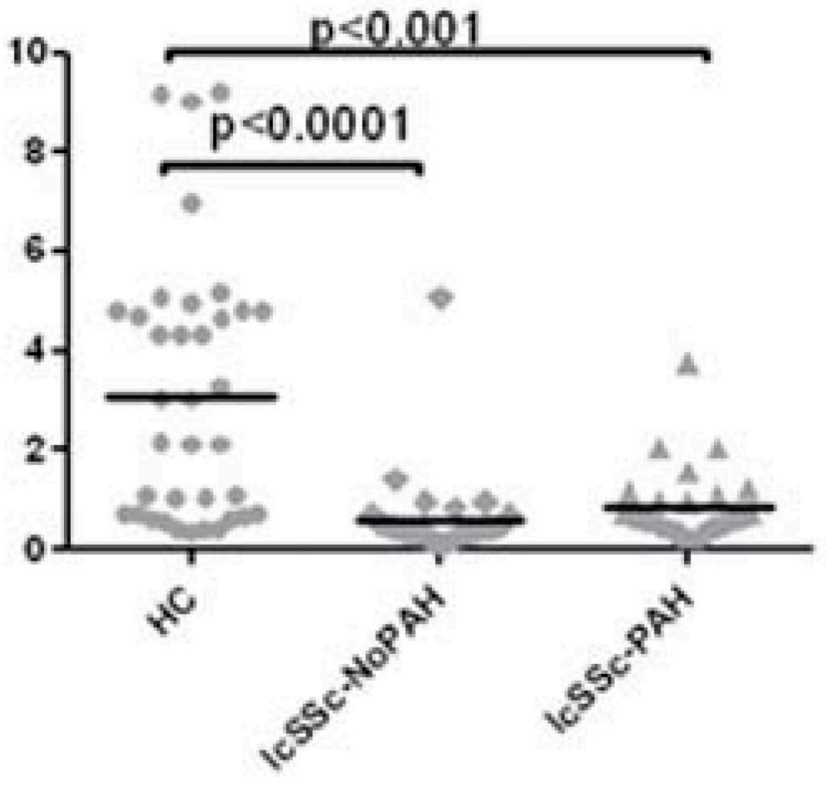

Figure 4. mRNA expression of IFN-regulated genes in patients with lcSSc PBMCs were isolated from HC $(n=36)$, lcSSc-NoPAH $(n=34)$ and lcSSc-PAH $(n=32)$. mRNA levels of IFIT1, IFIT2, IFITM1 and IRF4 were measured by qPCR. Expression of the housekeeping gene $\beta$-actin served as an internal positive control. Data are expressed as the fold-change normalized to mRNA expression in a single HC sample. Each data point represents a single subject; horizontal lines show the mean. 

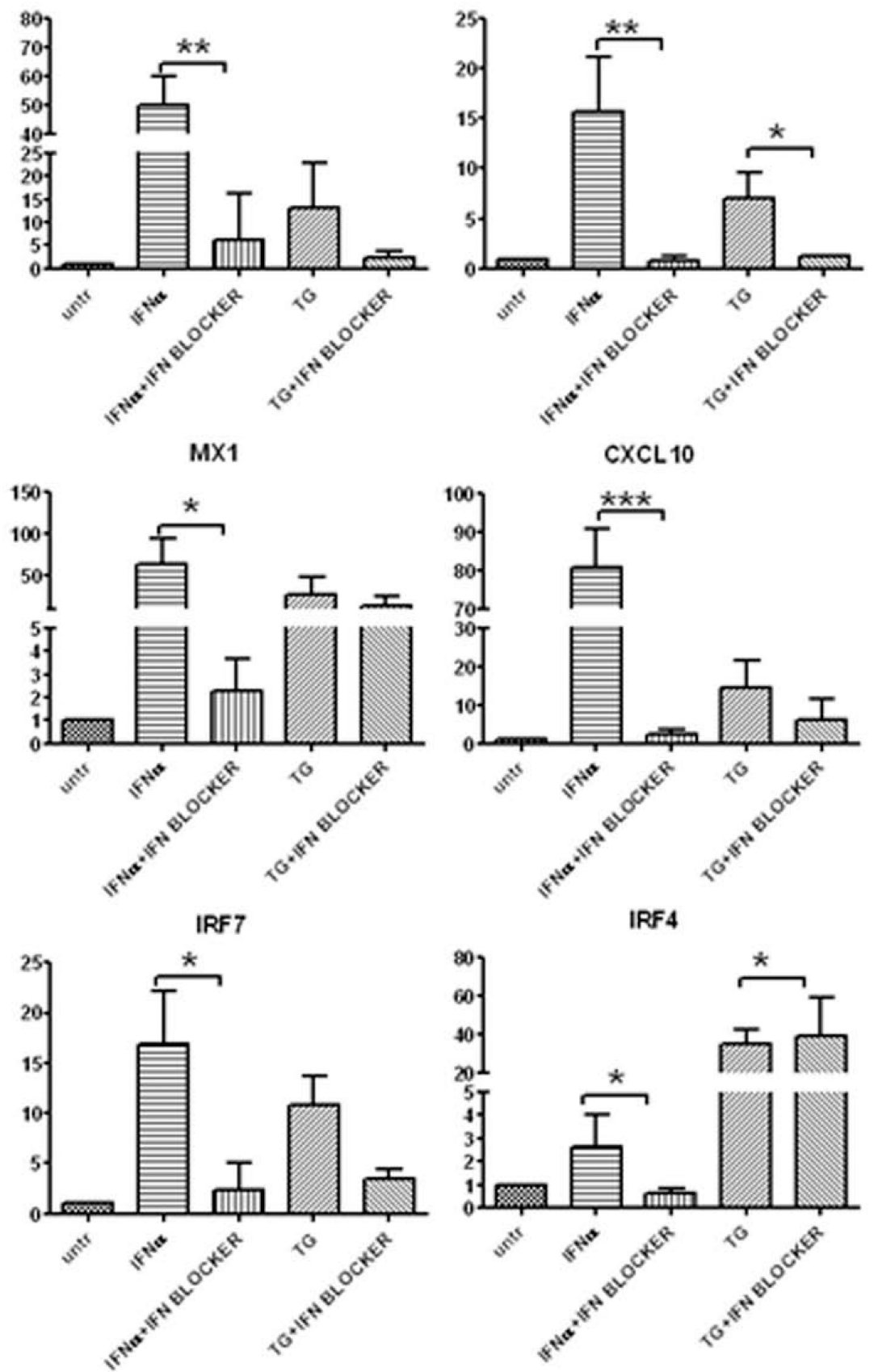

Figure 5. TG activates endogenous IFN and its target genes in HC PBMCs

PBMCs were isolated from HC and treated with IFNa (250U) or TG (5pM) for 24 hours in combination with B18R Recombinant protein (IFN blocker). mRNA levels of IFIT1, IFITM1, MX1, CXCL10, IRF7, and IRF4 were measured by qPCR. Expression of the housekeeping gene $\beta$-actin served as an internal positive control. Data are expressed as the fold-change normalized to mRNA expression in untreated HC PBMC. 

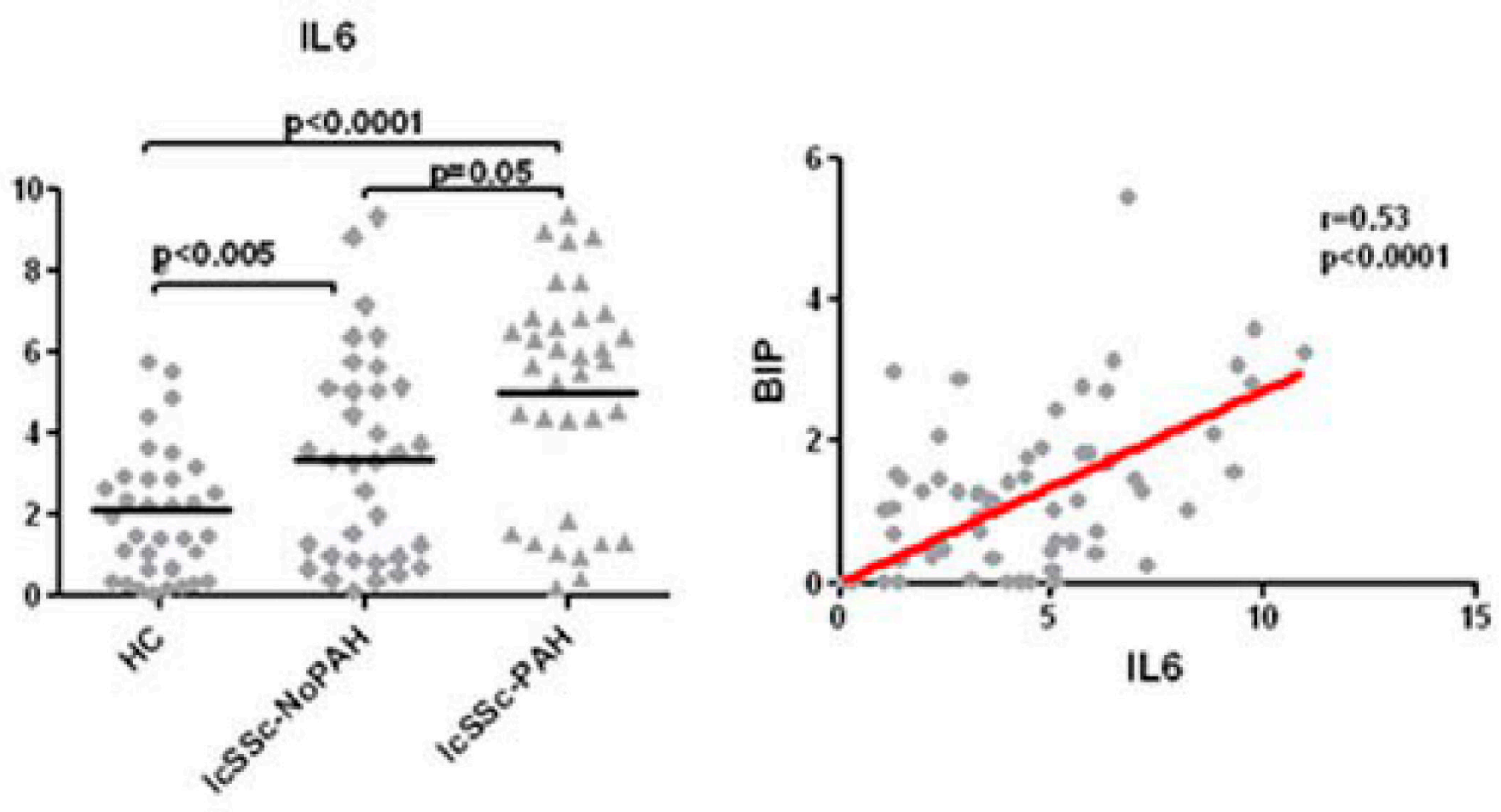

Figure 6. IL-6 expression correlates with ER stress/UPR genes mRNA levels of IL-6 was determined by qPCR in PBMC isolated from HC $(n=36)$, lcSScNoPAH $(n=34)$ and lcSSc-PAH $(n=32)$. Expression of the housekeeping gene $\beta$-actin served as an internal positive control. Data are expressed as the fold-change normalized to mRNA expression in a single $\mathrm{HC}$ sample. Each data point represents a single subject; horizontal lines show the mean. Right panel depicts linear regression analysis of the relationship between expression of BiP and IL-6 in PBMCs from ISSc patients. 